\title{
Fractional exhaled nitric oxide (FeNO) among office workers in an academic institution, Malaysia - associations with asthma, allergies and office environment
}

\begin{abstract}
Objective: There are few studies on fractional exhaled nitric oxide (FeNO) and respiratory symptoms among adults in tropical areas. The aim was to study associations between FeNO and selected personal factors, respiratory symptoms, allergies, office characteristics and indoor office exposures among office workers (n国 4 (46) from a university in Malaysia. Methods: Information on health was collected by a questionnaire, skin prick test and FeNO measurement. Temperature, relative air humidity, carbon monoxide and carbon dioxide were measured in the offices. Settled dust was vacuumed in the offices and analyzed for endotoxin, $(1,3)$-6-glucan and house dust mites allergens, namely Dermatophagoides pteronyssinus (Der p 1) and Dermatophagoides farinae (Der f 1). Two-level linear mixed models and multiple logistic regression were used to analyze the associations. Results: One-fourth (25.9\%) of the office workers had elevated FeNO level (Ó25 फppb) and 61.5\% had HDM, cat, seafood or

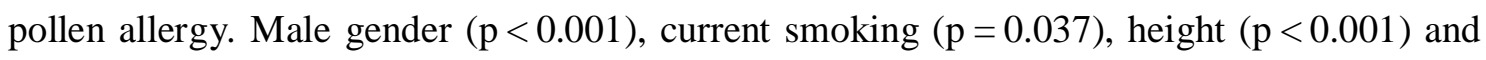
atopy $(\mathrm{p} \mathbb{R}[\mathrm{d} .001)$ were associated with FeNO. The amount of vacuumed dust was associated with FeNO among atopic subjects (p国圆.009). Asthma and rhinitis symptoms were associated

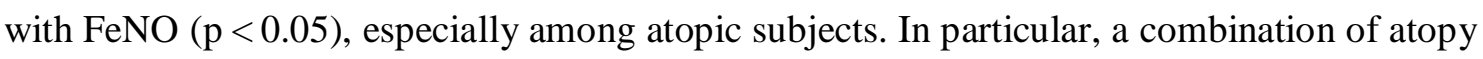
and elevated FeNO were associated with doctor-diagnosed asthma (p\&l001), rhinitis

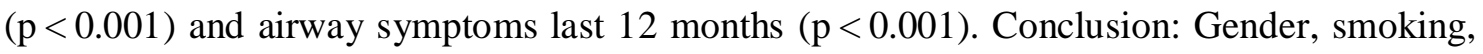
height and atopy are important risk factors for elevated FeNO levels. A combination of allergy testing and FeNO measurement could be useful in respiratory illness epidemiology studies and patient investigations in tropical areas.
\end{abstract}

Keyword: Adults; Allergy; Malaysia; Office; Respiratory symptoms; Rhinitis; Tropical areas 